\title{
Effect of sport-tinted contact lenses for contrast enhancement on retinal straylight measurements
}

\author{
Alejandro Cerviño ${ }^{1}$, Jose M. Gonzalez-Meijome ${ }^{2}$, João M. M. Linhares ${ }^{2}$, \\ Sarah L. Hosking ${ }^{3,4,5}$ and Robert Montes-Mico ${ }^{1}$ \\ ${ }^{1}$ Optics Department, Universidad de Valencia, Dr Moliner 50, 46100 Burjassot, Spain, ${ }^{2}$ Department \\ of Physics (Optometry), University of Minho, Portugal, ${ }^{3}$ Department of Optometry and Vision \\ Sciences, City University, UK, ${ }^{4}$ School of Life and Health Sciences, Aston University, UK and \\ ${ }^{5}$ Department of Ophthalmology, University of Melbourne, Australia
}

\begin{abstract}
Purpose: To investigate the effect of two tinted contact lenses (CL) designed for outdoor sports activity on the psychometric determination of retinal straylight using the compensation comparison method.

Methods: Thirteen emmetropic subjects were randomly fitted with two different tinted Nike Maxsight ${ }^{\circledR}$ (Bausch \& Lomb, Rochester, NY, USA) CL in one eye, while the contralateral eye was fitted with a clear lens made of the same material (Optima 38, Bausch \& Lomb). Three valid straylight measurements were taken on each eye before and a few minutes after lens insertion, when lens stabilization had occurred.

Results: The subjects' mean straylight values were $0.90 \pm 0.09$ at baseline and $0.95 \pm 0.10$ with the clear Optima $38 \mathrm{CL}$. Straylight values were $0.97 \pm 0.10$ and $1.0 \pm 0.10 \mathrm{log}$ units with the amber and grey-green tinted CL, respectively. Differences in straylight between baseline (without $\mathrm{CL}$ ) and with the clear $C L$ in place were neither statistically significant $(p=0.066)$ nor was there a significant difference between baseline and the amber CL $(p=0.052)$. However, the grey-green CL showed a statistically significant difference from baseline $(p=0.006)$. Differences in straylight with the clear $\mathrm{CL}$ compared with the grey-green CL were also statistically different from zero $(p=0.002)$ showing an increased straylight value for the tinted CL. These differences were variable, but consistent for each subject, thus those showing higher or lower changes with one tinted lens tended to show the same trend with the second lens $\left(r^{2}=0.736\right)$.

Conclusions: Despite increases having been found in straylight values with tinted contact lenses, those changes are not likely to induce clinically significant changes in visual function under photopic conditions, even for the grey-green $\mathrm{CL}$, which seems to increase straylight values more significantly than the amber $\mathrm{CL}$. This difference between the tinted $\mathrm{CL}$ could suggest a wavelength dependence of straylight values, although this should be investigated further by controlling for pupil size and subjects' pigmentation, as well as by using neutral density filters.
\end{abstract}

Keywords: contact lens, ocular scatter, polymacon, retinal straylight, tinted lens

Received: 19 October 2007

Revised form: 26 November 2007, 12 December 2007

Accepted: 14 December 2007

Correspondence and reprint requests to: Alejandro Cerviño.

Tel.: + 34963544 042; Fax: + 34963544715 .

E-mail address: alejandro.cervino@uv.es

\section{Introduction}

The retinal image can be degraded by many factors, including forward light scattering arising from the loss of transparency in the ocular media. Intraocular scattered light is light that has been reflected, refracted, diffracted or experienced multiple combinations of all three along the optical path of travel (Bohren, 1995). 
There are five major sources that contribute to the total amount of this ocular straylight: the cornea, the iris, the sclera, the retina and the lens (de Waard et al., 1992). It is assumed that for young healthy eyes the total amount of straylight is given $1 / 3$ by the cornea, $1 / 3$ by the lens and $1 / 3$ by sclera and retina, but these ratios change with age, pigmentation and specific pathologies. Corneal light scatter is constant with age (Ijspeert et al., 1990; van den Berg, 1995) while retinal straylight may also increase with lens opacities (i.e. cataracts) (Ijspeert et al., 1990) or after corneal refractive surgery (Veraart et al., 1992, 1993, 1995). The iris and the sclera scatter light depending on the patient's pigmentation (van den Berg et al., 1991); for example, brown eyes absorb more light and consequently produce less scatter than light eyes. Lens scatter increases with age resulting in greater straylight in patients with cataracts (de Wit et al., 2006). Finally, the retina produces light scatter in different locations depending on pigmentation (van den Berg et al., 1991).

A clinical application of straylight measurement is to diagnose patients with complaints, because of the associated contrast loss in the final retinal image, caused by a high degree of light scattering within the eye. Several clinical devices have been developed to evaluate straylight and glare (van Rijn et al., 2005). A recent computer version of the straylight meter has been designed to improve the clinical measurement of the ocular straylight (Franssen et al., 2006). This instrument has proven to be sensitive for systematic application in research and clinical activities providing repeatable measures of retinal straylight (Cervino et al., 2008).

Another field of application of straylight measurements is that of the ocular response to contact lens wear, particularly the evaluation of oedema (Elliott et al., 1991, 1993). Tinted contact lenses could therefore affect straylight, even in the absence of significant levels of oedema.

The new Nike Maxsight ${ }^{\circledR}$ contact lens (Polymacon, Bausch \& Lomb, Rochester, NY, USA) is a soft contact lens $(\mathrm{CL})$ described by the manufacturer as designed to improve the contrast of images and mainly aimed at those participating in sport outdoors. There are two different tints: amber (AM) and grey-green (GGM), both having different absorption spectra, as shown in Figure 1. If any significant increase in retinal straylight were present, this effect could mask the intended effect of contrast improvement. Retinal straylight has been shown to be highly dependent on age and pigmentation (Ijspeert et al., 1990), and furthermore, the effect of wavelength has been reported to depend directly on the level of pigmentation (Coppens et al., 2006). This therefore implies that some individual variability in retinal straylight for a given wavelength would be

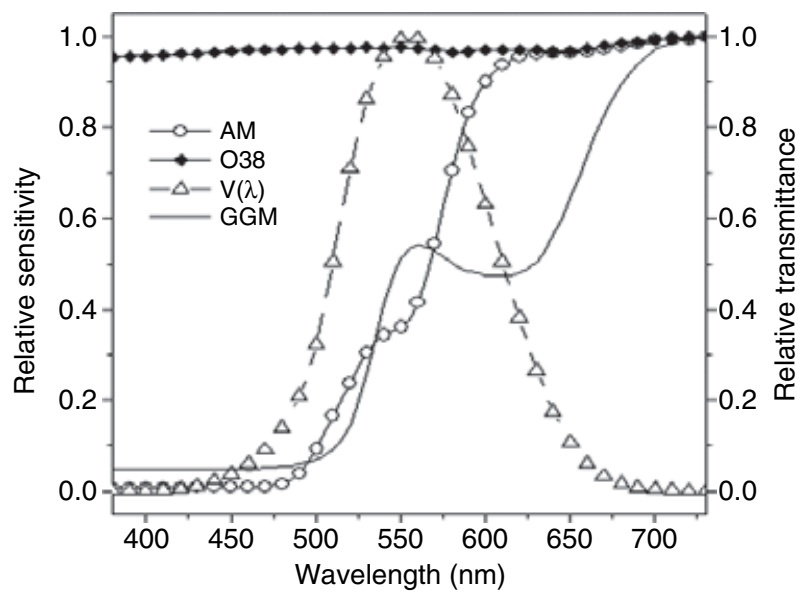

Figure 1. Normalized $\mathrm{CIE}$ photopic spectral luminous efficiency function (open-triangles line, adapted from Commission Internationale de l'Eclairage (CIE) (2004) plotted against the normalized mean transmittance of two AM (open-circles line) and two GGM (solid line) $\mathrm{CL}$, and the transmittance of $\mathrm{O} 38 \mathrm{CL}$ (black diamonds line).

expected because of the variability in pigmentation of the different individuals.

The aim of the present study was to investigate the effect of these sport-tinted contact lenses on retinal straylight in order to elucidate potential limitations of their use in normal viewing conditions.

\section{Materials and methods}

Thirteen emmetropic volunteers were recruited for the study (eight men, five women; mean age $24.2 \pm 3.3$ years). Refractive and ocular examination was carried out before inclusion. Contact lens wearers were excluded because of the potential confounding effect of chronic corneal odema. After the nature of the study was explained, a signed consent form was obtained from each subject. The study protocol followed the tenets of the Declaration of Helsinki and was reviewed and approved by the Scientific Committee of the University of Minho.

\section{Determination of retinal straylight}

The measurement of retinal straylight was done monocularly, with scotopic room conditions to avoid external light interfering with the measurements, using the C-Quant retinal straylightmeter (Oculus Optikgeräte $\mathrm{GmbH}$, Wetzlar-Dutenhofen, Germany), following the procedure described in detail elsewhere (Franssen et al., 2006), In brief, this system uses the compensation comparison method, a modification of the direct comparison method to obtain retinal straylight measures in a clinical setting. A flickering annulus is presented around a test field. This test field is divided into two 
halves. If there is intraocular scatter of the light from the annulus, these two halves will be perceived as flickering, but each will have a different modulation because one of them has counterphase compensation light added. The subject fixates and identifies in which of the two halves the flicker is stronger. A psychometrical response curve is computed from the responses obtained from the subject. As described elsewhere (Franssen et al., 2006), the examination process consists of two phases: a dark phase and a bright phase (maximum light intensity of the straylight source). The first phase has a dual purpose: to give a first estimate of the psychometric function, and therefore of the straylight value, and to be a training phase for the flicker comparison task, which will be more difficult as the straylight source (flickering annulus) becomes brighter. The second phase serves to refine this first estimate and gives the final straylight value.

\section{Maxsight ${ }^{\circledR}$ tinted contact lenses}

Maxsight ${ }^{\circledR}$ tinted contact lenses (polymacon) have recently appeared on the market, designed for outdoor sports activity by adding tints that theoretically filter light selectively to improve contrast for certain activities. Specifically, the two lenses used in this study are grey/ green, described by the manufacturer as designed for golf practice, and AM, for increasing contrast under fog or sunset conditions. There is currently very little scientific information regarding these lenses. A very recent report on the visual performance of football players with AM-tinted contact lenses compared with clear contact lenses showed that, in a sample of 35 eyes from 35 football players, contrast sensitivity was significantly improved with the Amber Maxsight ${ }^{\circledR}$ tinted contact lenses, although whether this was clinically significant was questioned by the author (Porisch, 2007). No information was provided on the clear lenses used, which seemed to be quite varied and this could have affected the results obtained.

\section{Procedure}

Baseline measurements were carried out prior to lens insertion with the C-Quant. Subjects were fitted in one eye with a clear hydrophilic CL Optima ${ }^{\circledR} 38$ (O38) and in the other eye with two tinted hydrophilic CL Nike Maxsight $^{\circledR}, A M$ and GGM, all made of the same material and manufactured by Bausch \& Lomb Inc. The assignment of a clear or tinted lens to the right or left eye was made according to a randomization table. The order in which each tinted lens was applied (to the same eye) was also randomized. Further technical details of the CL used in the study are provided in Table 1. Figure 1 provides details on the light transmittance of the clear and tinted contact lenses used as measured with a scanning spectrophotometer (UV-3101PC Shimadzu Corporation, Kyoto, Japan) along with the spectral luminous efficiency function of the human eye for photopic vision (adapted from Commission Internationale de l'Eclairage (CIE) (2004). After lens insertion, it was allowed to stabilize for 5 min. Retinal straylight was measured three times on each eye for each experimental condition.

Data were analyzed using the statistical package sPss version 14.0 (SPSS Inc., Chicago, IL, USA). Normal distribution of variables was assessed by the Kolmogorov-Smirnov normality test and homogeneity of variances was assessed by the Levene test. Differences between values obtained within the same session and between different sessions were assessed by independent $t$-test against zero. Differences between $\log$ values obtained under different experimental conditions were assessed statistically by paired $t$-test and Wilcoxon non-parametric test for variables with normal and non-normal distribution, respectively. Bi-variate correlations between the straylight values obtained with the different contact lenses against baseline were assessed by parametric (Pearson) and non-parametric (Spearman) correlations depending on the normal or non-normal distribution, respectively, of the variables involved. Regression analysis was also used in order to graphically illustrate the nature of those correlations. The level of statistical significance was established at $\alpha=0.05$.

\section{Results}

Descriptive statistics of the straylight values obtained with the different lenses are shown in Table 2, with repeated measures giving S.D. lower than 0.07 in all cases. To illustrate the increase in retinal straylight with the different contact lenses, the mean values and S.D. are shown in Figure 2.

Distribution was normal for the values obtained for baseline, and with the O38- and AM-tinted CLs but not for the GGM CL. Therefore, non-parametric tests were

Table 1. Technical details of the lenses used in the study

\begin{tabular}{lll}
\hline & Optima 38 & Nike Maxsight $^{\circledR}$ \\
\hline Manufacturer & Bausch \& Lomb & Bausch \& Lomb \\
Material (USANC) & Polymacon & Polymacon \\
Manufacturing & Spin-cast/lathe-cut & Spin-cast/lathe-cut \\
Hydration (FDA) & $38.6 \%$ (group I) & $38.6 \%($ group I) \\
Dk & 8.5 barrer & $8.5 \mathrm{barrer}$ \\
BC & $8.7 \mathrm{~mm}$ & $8.7 \mathrm{~mm}$ \\
Diameter & $14.2 \mathrm{~mm}$ & $14.3 \mathrm{~mm}$ \\
Power & -0.25 & -0.25 \\
Tint & No & Amber/grey-green \\
\hline
\end{tabular}


Table 2. Descriptive statistics of straylight values obtained on thirteen eyes.

\begin{tabular}{|c|c|c|c|c|}
\hline & \multirow[b]{2}{*}{ Mean } & \multirow[b]{2}{*}{ S.D. } & \multicolumn{2}{|c|}{$\begin{array}{l}95 \% \text { Confidence } \\
\text { Interval }\end{array}$} \\
\hline & & & Upper & Lower \\
\hline Baseline & 0.902 & 0.086 & 1.071 & 0.732 \\
\hline Optima 38 & 0.950 & 0.100 & 1.146 & 0.754 \\
\hline Amber Maxsight ${ }^{\circledR}$ & 0.967 & 0.103 & 1.170 & 0.764 \\
\hline Grey/green Maxsight ${ }^{\circledR}$ & 0.999 & 0.098 & 1.191 & 0.808 \\
\hline
\end{tabular}

Values are presented as logarithmic units of straylight.

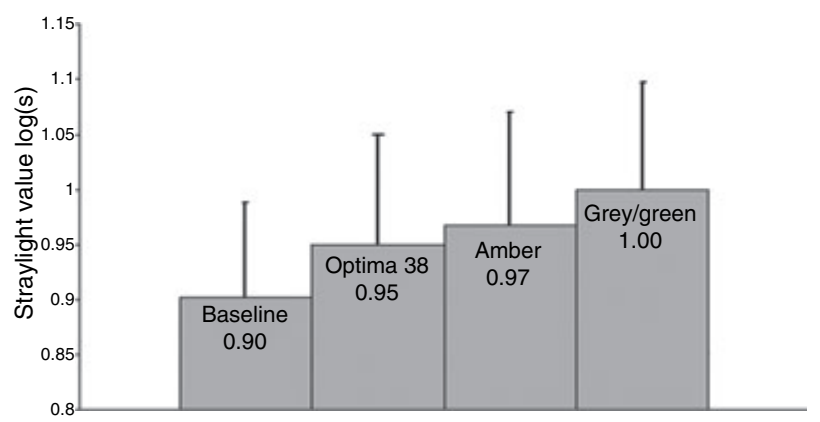

Figure 2. The descriptive statistics of Table 2 are illustrated for each experimental condition. Note the increase in the mean straylight value from baseline with all tested contact lenses. Values are presented as logarithmic units of straylight [log(s)].

applied to analysis involving values obtained for the GGM CL. Paired sample $t$-test did not show significant differences between the straylight baseline values and those obtained with the AM lens (mean difference: $-0.065 \pm 0.110 ; \quad p=0.052), \quad$ or with the $\mathrm{O} 38$ $(p=0.066)$. The Wilcoxon signed rank test did, however, show significant differences between the straylight baseline values and those obtained with the GGM CL (mean difference: $-0.098 \pm 0.091 ; p=0.006$ ).

Figure 3 shows the correlation between the differences from baseline straylight values with values obtained with AM and GGM Maxsight ${ }^{\circledR}$ tinted lenses. It shows that the changes in straylight induced by each lens are significantly correlated $\left(r^{2}=0.713\right.$; $p<0.01)$; thus, for example, subjects displaying small changes of straylight from baseline with one lens tend to show a similar change with the other tint; those experiencing a large change in straylight did so with both CLs.

Figure 4 represents the increments in straylight values from baseline with the clear lens and with both tinted lenses for each one of the thirteen subjects enrolled in the clinical study. Figure 2 suggests a progressive increase in straylight values from baseline, to O38, $\mathrm{AM}$ and GGM, with the latter presenting the higher degrees of scatter. Despite this general trend, Figure 4

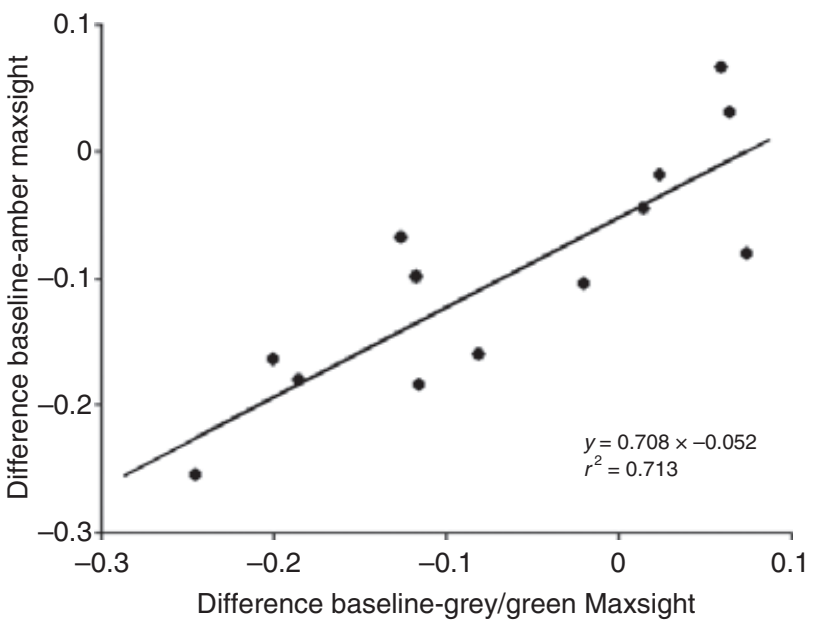

Figure 3. Correlation of differences in straylight values between baseline and those obtained with AM and GGM Maxsight ${ }^{\circledR}$ tinted contact lenses. Values are presented as logarithmic units of straylight $[\log (s)]$.

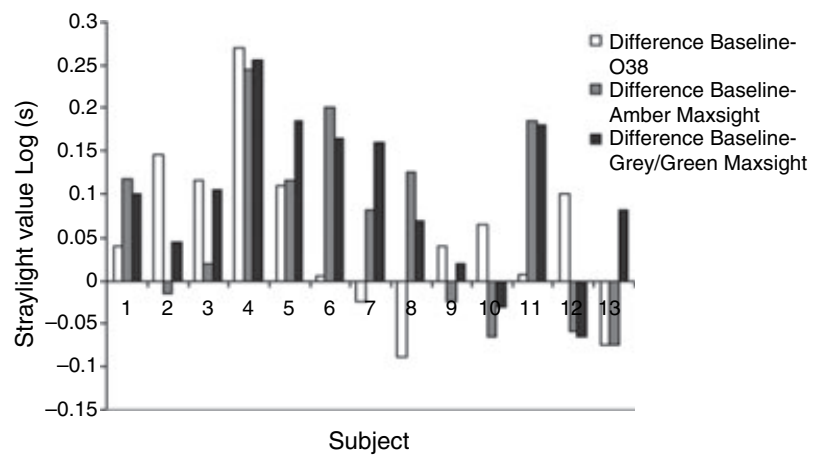

Figure 4. Mean difference from baseline in stray light value, $\log (\mathrm{s})$, for each of the 13 subjects when fitted with the O38-, and AM- and GGM-tinted lenses. Values are presented as logarithmic units of straylight $[\log (\mathrm{s})]$.

clearly shows that there is some variability at an individual level in the response to the $\mathrm{CL}$.

\section{Discussion}

The compensation comparison method has been shown to be a repeatable and reliable tool for the assessment of retinal straylight. In a previous study describing and assessing the procedure used here, Franssen et al. (2006) found an overall S.D. of repeated measures between 0.06 and $0.1 \log$ units, recently confirmed by Cervino et al. (2008), and this repeatability is not affected by the age of the subject. These S.D. values agree with those obtained in the present study.

Measurement of retinal straylight has been shown to be highly dependent on age and pigmentation (Coppens et al., 2006), and would clearly be affected by changes in 
corneal hydration because of contact lens wear (Elliott et al., 1991). For that reason, a change in retinal straylight following the insertion of tinted CL would be expected.

All the subjects examined in this study were emmetropic and they were not habitual CL wearers. Figure 4 shows the short-term mean effect of lens insertion in a non-contact lens wearer. The aim of the present study was to assess the variations in retinal straylight because of the tint of the Maxsight ${ }^{\circledR} \mathrm{CL}$. In order to avoid the influence of corneal hydration because of CL wear, a clear CL of the same material and parameters was fitted on the other eye and acted as a control. The response differed between subjects, but the expected increase in the mean straylight values following the insertion of all three types of lenses was observed.

One of the limitations of the present study is the lack of control over pupil size. Psychometric determination of intraocular scatter using the compensation comparison approach is essentially a photopic method, and the different light flux through the different CL would suggest a different level of pupil response that might have an effect on the straylight values obtained because scattering is not uniform over the pupil. The results reported by Franssen et al. (2007) suggest, however, that straylight values measured with photopic pupils are also valid for mesopic and scotopic pupils. A second limitation of the study is the lack of objective measures of scatter induced by the CL itself using a scattermeter. This would have allowed stronger conclusions regarding the effect of each of the lenses to be drawn from the present study and to differentiate the contributions from the lens, the tint, pupil size differences, etc. on the final straylight values obtained.

The C-Quant straylightmeter was conceived to implement the psychometrical determination of retinal straylight in clinical settings, but this means that some limitations have to be accepted in order to make it practical in clinical terms. The C-Quant determines retinal straylight but assumes constant angular dependence. This should be considered for the interpretation of the results given in the present study, because for large scatter angles and small pupil diameters a significant contribution to straylight is given by eye wall translucency depending on wavelength and pigmentation (Franssen et al., 2007).

This study has shown that sport-tinted contact lenses can significantly affect the straylight measurement compared with baseline and the use of a clear contact lens. Straylight can be modelled as a sum of three components (Coppens et al., 2006): a base straylight, which is strongly wavelength dependent, and then two components which depend on pigmentation and age. Corneal light scattering is considered to be the same for all healthy individuals and ages (van den Berg and Tan,
1994). In the present study, we have basically modified this value by adding a tinted CL. Coppens et al. (2006) have shown a very strong wavelength dependence of retinal straylight values obtained in young and wellpigmented eyes, which did not occur in less pigmented eyes. The eyes examined in this study were all wellpigmented (grades 3-4 according to the grading system of Seddon et al. (1990), and therefore these results might not be the same if the sample included lesspigmented eyes.

Despite significant differences from baseline being found for the GGM lenses, these straylight differences do not seem likely to affect the quality of vision when considering contrast enhancement at a clinical level (Porisch, 2007), although because they are conceived for sport activity the implications may be significant at that level. The variations with pigmentation and age among the population in larger studies could, however, reveal different results. Further studies should be carried out to confirm this hypothesis and to evaluate the subjective and objective impact of such lenses on visual performance under different illumination conditions. Furthermore, the variability observed in individual responses concerning tinted contact lenses, warrants further research in order to elucidate if such individual variations are linked to any interindividual spectral sensitivity differences to the pattern created by the C-Quant.

As nowadays many intraocular lenses use a tinted UV radiation filter, other fields of application for this test could be in the evaluation of post-surgical impact of retinal scatter on the visual performance of such lens types.

Figure 3 showed how the changes in straylight induced by the spectral lenses are consistent, as those subjects with low change with AM also tend to show low change with GGM while those with higher changes with AM also display higher changes with GGM lens. This suggests some interindividual susceptibility to experience more or less change which is consistent for both lenses. This agrees with the conclusions obtained by Porisch (2007) on contrast sensitivity with the AM compared with clear contact lenses.

The present report is, to the best of the authors' knowledge, the first contralateral controlled study addressing the influence of tinted contact lenses for outdoor sports, on the light scatter on the retina. The results reported in this study could evidence a wavelength dependence for straylight values, although this should be investigated further by controlling for pupil size and using neutral density filtres, as well as increasing sample size, and grouping subjects by pigmentation. It will also be interesting to confirm if some individual characteristics pre-dispose subjects to experience larger changes in straylight with certain tints, and which could be predictors of such behaviour. 


\section{Acknowledgments}

The authors wish to thank Bausch \& Lomb for providing the Nike Maxsight ${ }^{\circledR}$ contact lenses for the present study. The authors have no financial interest in any of the issues contained in this article and have no proprietary interest in the development or marketing of the products or medical equipment used in this study.

\section{References}

van den Berg, T. J. (1995) Analysis of intraocular straylight, especially in relation to age. Optom. Vis. Sci. 72, 52-59.

van den Berg, T. J. and Tan, K. E. (1994) Light transmittance of the human cornea from 320 to $700 \mathrm{~nm}$ for different ages. Vision Res. 34, 1453-1456.

van den Berg, T. J., Ijspeert, J. K. and de Waard, P. W. (1991) Dependence of intraocular straylight on pigmentation and light transmission through the ocular wall. Vision Res. 31, 1361-1367.

Bohren, C. (1995) Scattering by particles. In: Handbook of Optics, 2nd edn, (ed M. Bass), McGraw-Hill Publishing Co, New York, pp. 6-19.

Cervino, A., Montes-Mico, R. and Hosking, S. L. (2008) Performance of the compensation comparison method for retinal straylight measurement. Effect of patient's age on repeatability. Br. J. Ophthalmol. (in press)

Commission Internationale de l'Eclairage (CIE) (2004) Colorimetry, CIE Publication 015:2004, 3rd edn. Central Bureau of CIE, Vienna, Austria.

Coppens, J. E., Franssen, L. and van den Berg, T. J. (2006) Wavelength dependence of intraocular straylight. Exp. Eye Res. 82, 688-692.

Elliott, D. B., Mitchell, S. and Whitaker, D. (1991) Factors affecting light scatter in contact lens wearers. Optom. Vis. Sci. 68, 629-633.

Elliott, D. B., Fonn, D., Flanagan, J. and Doughty, M. (1993) Relative sensitivity of clinical tests to hydrophilic lensinduced corneal thickness changes. Optom. Vis. Sci. 70, 1044-1048.
Franssen, L., Coppens, J. E. and van den Berg, T. J. (2006) Compensation comparison method for assessment of retinal straylight. Invest. Ophthalmol. Vis. Sci. 47, 768-776.

Franssen, L., Tabernero, J., Coppens, J. E. and van den Berg, T. J. (2007) Pupil size and retinal straylight in the normal eye. Invest. Ophthalmol. Vis. Sci. 48, 2375-2382.

Ijspeert, J. K., de Waard, P. W., van den Berg, T. J. and de Jong, P. T. (1990) The intraocular straylight function in 129 healthy volunteers; dependence on angle, age and pigmentation. Vision Res. 30, 699-707.

Porisch, E. (2007) Football players' contrast sensitivity comparison when wearing amber sport-tinted or clear contact lenses. Optometry 78, 232-235.

van Rijn, L. J., Nischler, C., Gamer, D., Franssen, L., de, W. G., Kaper, R., Vonhoff, D., Grabner, G., Wilhelm, H., Volker-Dieben, H. J. and van den Berg, T. J. (2005) Measurement of stray light and glare: comparison of Nyktotest, Mesotest, stray light meter, and computer implemented stray light meter. Br. J. Ophthalmol. 89, 345351.

Seddon, J. M., Sahagian, C. R., Glynn, R. J., Sperduto, R. D. and Gragoudas, E. S. (1990) Evaluation of an iris color classification system. The Eye Disorders Case-Control Study Group. Invest. Ophthalmol. Vis. Sci. 31, 1592-1598.

Veraart, H. G., van den Berg, T. J., Ijspeert, J. K. and Cardozo, O. L. (1992) Stray light in radial keratotomy and the influence of pupil size and straylight angle. Am. J. Ophthalmol. 114, 424-428.

Veraart, H. G., van den Berg, T. J., Hennekes, R. and Adank, A. M. (1993) Stray light in photorefractive keratectomy for myopia. Bull. Soc. Belge Ophtalmol. 249, 57-62.

Veraart, H. G., van den Berg, T. J., Hennekes, R. and Adank, A. M. (1995) Stray light in photorefractive keratectomy for myopia. Doc. Ophthalmol. 90, 35-42.

de Waard, P. W., Ijspeert, J. K., van den Berg, T. J. and de Jong, P. T. (1992) Intraocular light scattering in age-related cataracts. Invest. Ophthalmol. Vis. Sci. 33, 618-625.

de Wit, G. C., Franssen, L., Coppens, J. E. and van den Berg, T. J. (2006) Simulating the straylight effects of cataracts. J. Cataract Refract. Surg. 32, 294-300. 